\title{
Stress and burnout in residents: impact of mindfulness-based resilience training
}

This article was published in the following Dove Press journal:

Advances in Medical Education and Practice

25 August 2015

Number of times this article has been viewed

\author{
Brian E Goldhagen' \\ Karen Kingsolver ${ }^{2}$ \\ Sandra S Stinnett' \\ Jullia A Rosdahl' \\ 'Department of Ophthalmology, \\ ${ }^{2}$ Department of Family and \\ Community Medicine, Duke \\ University Medical Center, Durham, \\ NC, USA
}

Background and objective: Stress and burnout impact resident physicians. This prospective study tests the hypothesis that a mindfulness-based resilience intervention would decrease stress and burnout in residents.

Methods: Resident physicians from the Departments of Family Medicine, Psychiatry, and Anesthesia at Duke University, Durham, NC, USA, participated in two or three 1-hour sessions of mindfulness-based resilience activities, which introduced mindful-awareness and included practical exercises for nurturing resilience. Anonymous surveys were distributed before (completed by 47 residents) and after the intervention (both completed by 30 residents); a follow-up survey was distributed 1 month later (seven residents completed all three surveys). The survey included the Depression Anxiety Stress Scale, 21-question version (DASS-21), the Oldenburg Burnout Inventory, the Mindful Attention Awareness Scale, and ten questions from the Cognitive Failures Questionnaire.

Results: At baseline, most residents' scores were in the normal range with respect to stress; however, female residents had higher DASS-21 scores than male residents $(31.7$, females vs 18.4 , males; $P=0.002$ ). Most residents' burnout scores were in the abnormal range, both with respect to exhaustion $(38 / 47$ residents, subscore $\geq 2.25$ ) and disengagement (37/47 residents, subscore $\geq 2.1$ ). Higher perceived levels of stress correlated with the instruments. Analysis of the surveys before and after the intervention showed no significant short-term change in stress, burnout, mindful-awareness, or cognitive failure. There was a trend for females and post-medical school graduate year 1 and 2 (PGY1 and PGY2) residents to have a reduction in DASS-21 scores after intervention. There was also a trend of reduced stress and burnout in residents who perceived higher stress.

Conclusion: Residents who are female, PGY1 and PGY2, and who perceive residency to be stressful may benefit most from a mindfulness-based resilience intervention.

Keywords: physician, self-care, wellness, medical trainees

\section{Introduction}

Stress and burnout among resident physicians have implications for patient care and the health and well-being of our professional community. Sources of such stress in resident training can be divided into three components: 1) situational (workload, sleep deprivation, and poor learning environment); 2) personal (family, isolation, and financial); and 3) professional (overwhelming patient responsibility and information). ${ }^{1-3}$ Stress resulting from the discrepancy between the individual's ideals or expectations and the reality of occupational life is a source of burnout. ${ }^{4}$ Burnout is a "syndrome of emotional exhaustion, depersonalization, and reduced personal accomplishment", and can lead to negative repercussions for the individual experiencing burnout, the
Correspondence: Jullia A Rosdahl Duke Eye Center, Duke University Medical Center 235I Erwin Road, DUMC 3802, Durham, NC 277I0, USA

Tel + I 9196848850

Fax +19196818267

Email jullia.rosdahl@duke.edu 
people for whom care is provided, and for the community at large. ${ }^{6}$ Higher levels of burnout among residents are associated with perceived suboptimal patient care and decreased effectiveness. ${ }^{7-10}$

Therefore, adding stress management training to the medical education curriculum could help the trainee to deal more successfully with the stress of training, develop techniques to help in his or her career, and potentially prevent physician burnout. Mindfulness-based stress reduction has been demonstrated to help individuals more effectively manage stress, pain, and other health conditions. ${ }^{11}$ Mindfulness is defined as "the awareness that arises from paying attention on purpose, in the present moment, nonjudgmentally". ${ }^{12}$ Since medical students demonstrated decreased stress and depression when stress management was integrated into medical student education, ${ }^{13}$ this type of program may be helpful to residents. However, integrating an intensive 8-week mindfulness-based stress reduction program into a residency program is usually unfeasible for logistic reasons including clinical needs of the service, competing educational priorities, and lack of time and money.

For our current study, we developed a short practical mindfulness-based intervention for residents, and assessed the short-term effect of the intervention on stress and burnout in residents. Our hypothesis was that a mindfulness-based intervention would decrease the stress and burnout in resident physicians. In addition, a secondary exploratory analysis of correlations between demographics and stress or burnout was undertaken to help identify at-risk residents.

\section{Methods}

Ethics approval for the current prospective study was granted by the Duke University Medical Center's Institutional Review Board, and the present study was conducted in accordance with the standards of the Health Insurance Portability and Accountability Act. A previous pilot study with residents from the Department of Ophthalmology was used for sample size calculation: a sample size of 26 residents was needed for $90 \%$ power to detect a difference in mean stress scores of 1.2, between two points (standard deviation 1.79, alpha 0.05). ${ }^{14}$ The current study (performed in 2012 and 2013) included resident physicians from the Departments of Family Medicine, Psychiatry, and Anesthesia at Duke University, Durham, NC, USA. These residency programs were selected based on the program directors' interest in offering their residents the mindfulness-based resilience training. The resilience training was developed by a trained clinical psychologist (KK) and was offered to residency programs throughout the university.
Participation in the present study was voluntary and anonymous; the option to participate in the study was given at the start of the mindfulness-based resilience training sessions. Participation in the study was not required for participation in the training sessions. Surveys were distributed just prior to the first session and just after the last session (2-8 weeks apart, based on program scheduling). Follow-up online surveys were distributed 1 month following the final session (with one email reminder), using Research Electronic Data Capture (REDCap). ${ }^{15}$ Surveys were matched using a combination of three identifiers (favorite color, first car, and school mascot). Demographic information included sex, age, and number of children, training specialty, and post-medical school graduate year (PGY) level. The survey consisted of 60 questions total and included validated survey instruments: the Depression-Anxiety-Stress Scale, 21-question version (DASS-21), ${ }^{16,17}$ the Oldenburg Burnout Inventory (OLBI), ${ }^{18,19}$ the Mindful Attention Awareness Scale (MAAS), ${ }^{20}$ and ten questions from the Cognitive Failures Questionnaire (CFQ). ${ }^{21}$ Abnormally high values on the OLBI were $\geq 2.25$ on the exhaustion scale and $\geq 2.1$ on the disengagement scale. ${ }^{22}$ Residents were asked about their perceived level of stress during residency. A copy of the survey is available upon request.

The intervention consisted of two or three 1-hour mindfulness-based resilience training sessions. These group sessions were developed and presented by a clinical psychologist with expertise in mindfulness-based techniques, who introduced the concepts of mindful-awareness and used practical exercises that emphasized mediation, value exploration, and cultivation of positivity to nurture resilience. Sessions were held during the protected resident didactic time.

Statistical analysis was performed using SAS software, version 9.2 (SAS Inc., Cary, NC, USA). A $P$-value $<0.05$ was considered statistically significant. Baseline instrument scores were compared by demographic group, using the Kruskal-Wallis test, to look for differences in stress and burnout. Similarly, the instrument scores were compared among the responses to the single question about perceived stress to see how this single question related to the instrument scores. To examine the effect of the intervention within the group of residents who completed both surveys, the instrument scores of those residents were compared at two timepoints using the Wilcoxon signed-rank test. To determine if our intervention was helpful to specific subsets of residents, a difference in means was calculated for each of the residents who completed both surveys to quantify the change in scores over the study period; these values were compared 
Table I Baseline stress, burnout, mindful attention awareness, and cognitive failure scores

\begin{tabular}{|c|c|c|c|c|c|}
\hline & $\begin{array}{l}\text { DASS total } \\
\text { mean (SD) }\end{array}$ & $\begin{array}{l}\text { OLBI } \\
\text { exhaustion subscore } \\
\text { mean (SD) }\end{array}$ & $\begin{array}{l}\text { OLBI } \\
\text { disengagement subscore } \\
\text { mean (SD) }\end{array}$ & $\begin{array}{l}\text { MAAS } \\
\text { mean (SD) }\end{array}$ & $\begin{array}{l}\text { Cognitive } \\
\text { failures score } \\
\text { mean (SD) }\end{array}$ \\
\hline \multicolumn{6}{|l|}{ Age, years } \\
\hline $20-29(n=25)$ & $23.0(16.5)$ & $2.5(0.5)$ & $2.4(0.5)$ & $3.8(0.8)$ & I7.8 (7.3) \\
\hline \multirow[t]{2}{*}{$30-39(n=22)$} & $28.4(17.1)$ & $2.6(0.4)$ & $2.4(0.5)$ & $3.7(0.8)$ & $17.4(5.0)$ \\
\hline & $P=0.236$ & $P=0.707$ & $P=0.966$ & $P=0.956$ & $P=0.877$ \\
\hline \multicolumn{6}{|l|}{ Sex } \\
\hline Female $(n=25)$ & $31.7(15.1)$ & $2.6(0.3)$ & $2.4(0.4)$ & $3.6(0.8)$ & $19.9(6.3)$ \\
\hline \multirow[t]{2}{*}{ Male $(n=22)$} & $18.4(16.2)$ & $2.4(0.5)$ & $2.4(0.5)$ & $4.0(0.8)$ & I $4.8(5.0)$ \\
\hline & $P=0.002$ & $P=0.033$ & $P=0.805$ & $P=0.083$ & $P=0.008$ \\
\hline \multicolumn{6}{|l|}{ Children } \\
\hline No $(n=36)$ & $25.4(14.9)$ & $2.5(0.4)$ & $2.4(0.4)$ & $3.7(0.8)$ & $18.2(6.3)$ \\
\hline \multirow[t]{2}{*}{ Yes $(n=I I)$} & $25.6(22.8)$ & $2.4(0.5)$ & $2.4(0.6)$ & $4.0(0.7)$ & I5.6 (5.8) \\
\hline & $P=0.606$ & $P=0.487$ & $P=0.970$ & $P=0.486$ & $P=0.284$ \\
\hline \multicolumn{6}{|l|}{ Residency year } \\
\hline PGYI $(n=8)$ & $29.5(11.6)$ & $2.6(0.4)$ & $2.4(0.4)$ & $3.8(1.0)$ & $22.9(9.0)$ \\
\hline PGY2 $(n=16)$ & $22.6(16.3)$ & $2.5(0.4)$ & $2.3(0.4)$ & $3.8(0.8)$ & I6.5 (4.7) \\
\hline PGY3 $(n=14)$ & $26.9(17.6)$ & $2.5(0.6)$ & $2.5(0.5)$ & $3.6(0.8)$ & $16.3(6.6)$ \\
\hline \multirow[t]{2}{*}{ PGY4 $(n=7)$} & $18.6(16.5)$ & $2.3(0.3)$ & $2.4(0.6)$ & $4.0(0.6)$ & I5.I (4.2) \\
\hline & $P=0.344$ & $P=0.333$ & $P=0.648$ & $P=0.689$ & $P=0.249$ \\
\hline \multicolumn{6}{|l|}{ Race/ethnicity } \\
\hline White $(n=28)$ & $23.6(15.0)$ & $2.5(0.4)$ & $2.4(0.4)$ & $3.8(0.8)$ & $16.6(6.2)$ \\
\hline Asian $(n=6)$ & $29.7(16.5)$ & $2.5(0.3)$ & $2.4(0.4)$ & $3.9(0.8)$ & $20.0(5.7)$ \\
\hline Hispanic $(n=3)$ & $16.0(11.1)$ & $2.1(0.5)$ & $2.0(0.3)$ & $3.8(0.4)$ & $17.0(5.0)$ \\
\hline Multiple $(n=4)$ & $26.5(24.4)$ & $2.5(0.5)$ & $2.3(0.7)$ & $4.0(1.0)$ & $15.3(7.1)$ \\
\hline \multirow[t]{2}{*}{ Other $(n=4)$} & $28.1(20.1)$ & $2.9(0.4)$ & $2.8(0.6)$ & $3.0(0.9)$ & $20.8(8.2)$ \\
\hline & $P=0.84 I$ & $P=0.244$ & $P=0.26 \mathrm{I}$ & $P=0.455$ & $P=0.600$ \\
\hline \multicolumn{6}{|l|}{ Specialty } \\
\hline Anesthesiology $(n=20)$ & $22.0(14.5)$ & $2.5(0.4)$ & $2.3(0.4)$ & $3.8(0.7)$ & $16.6(4.0)$ \\
\hline Family medicine $(n=9)$ & $24.7(20.2)$ & $2.5(0.5)$ & $2.4(0.7)$ & $4.0(1.0)$ & $15.6(5.5)$ \\
\hline \multirow[t]{2}{*}{ Psychiatry $(n=18)$} & $29.8(17.4)$ & $2.5(0.5)$ & $2.5(0.4)$ & $3.6(0.8)$ & $19.6(8.0)$ \\
\hline & $P=0.375$ & $P=0.890$ & $P=0.229$ & $P=0.307$ & $P=0.283$ \\
\hline
\end{tabular}

Notes: DASS-2I scores indicated females had more negative emotional states than males. High burnout (both OLBI exhaustion and OLBI disengagement) was seen among residents and was greater in females than in males. Female residents also had more cognitive failures than males. P-value based on Kruskal-Wallis test.

Abbreviations: PGY, post-medical school graduate year; DASS-2I, Depression-Anxiety-Stress Scale, 21 -question version; OLBI, Oldenburg Burnout Inventory; MAAS, Mindful Attention Awareness Scale; SD, standard deviation.

among the demographic groups using the Kruskal-Wallis test. Although the sample size was not powered for this secondary analysis, trends could suggest groups to target for this intervention.

\section{Results}

Forty-seven residents completed the initial survey: 20 of 41 (49\%) anesthesiology residents, nine of 12 (75\%) family medicine residents, and 18 of 56 (32\%) psychiatry residents.

Table 2 Baseline perceived stress level

\begin{tabular}{lllll}
\hline Do you find residency stressful? & $\begin{array}{l}\text { Occasionally } \\
\text { stressful } \mathbf{n = 5}\end{array}$ & $\begin{array}{l}\text { Somewhat } \\
\text { stressful } \mathbf{n = 2} \text { I }\end{array}$ & $\begin{array}{l}\text { Very stressful } \\
\mathbf{n = 2 0}\end{array}$ \\
\hline DASS-2I total, mean (SD) & $13.2(7.7)$ & $21.2(14.3)$ & $34.2(17.1)$ & 0.008 \\
Stress subscale & $6.4(4.6)$ & $10.8(6.3)$ & $17.1(9.0)$ & 0.009 \\
Anxiety subscale & $4.0(3.7)$ & $5.0(3.9)$ & $7.7(5.8)$ & 0.220 \\
Depression subscale & $2.8(2.3)$ & $5.4(7.5)$ & $9.4(6.8)$ & 0.016 \\
Oldenburg Burnout Inventory (OLBI) & $2.0(0.4)$ & $2.5(0.4)$ & $2.6(0.4)$ & 0.022 \\
Disengagement subscore & $2.0(0.4)$ & $2.4(0.5)$ & $2.5(0.4)$ & 0.064 \\
Exhaustion subscore & $2.0(0.5)$ & $2.5(0.4)$ & $2.6(0.4)$ & 0.013 \\
Mindful Attention Awareness Scale (MAAS) & $4.6(0.3)$ & $3.9(0.7)$ & $3.4(0.8)$ & $19.1(4.9)$ \\
Cognitive failures score & $11.2(4.8)$ & $17.7(6.9)$ & 0.022 \\
\hline
\end{tabular}

Notes: Study participants answered the question, "Do you find medical training and residency to be stressful?" The answer choices were: very stressful, somewhat stressful, occasionally stressful, or not stressful. None of the residents in this study chose "not stressful". Stress, burnout, mindful awareness, and cognitive failure scores are shown for the groups of residents who indicated each stress level. Higher perceived levels of stress correlated with higher stress scores on the DASS-2I, higher burnout scores on the OLBI, lower awareness of mindfulness on the MAAS, and greater cognitive failure scores. P-value based on Kruskal-Wallis test.

Abbreviations: DASS-2I, Depression-Anxiety-Stress Scale, 2 I-question version; SD, standard deviation; PGY, post-medical school graduate year. 
Thirty residents completed the two surveys, just prior to the first session and just following the final session; seven of those 30 residents completed the follow-up online survey.

\section{Baseline stress and burnout in resident physicians}

At baseline, most resident scores were in the normal range with respect to stress (31/47 residents), anxiety (29/47 residents), and depression (36/47 residents), using the DASS-21 questionnaire. Greater stress was measured in female residents compared with males at baseline. The DASS-21 total was 31.7 , females vs 18.4 , males $(P=0.002)$; the stress subscale was 15.9 females vs 9.3 males $(P=0.001)$; the anxiety subscale was 7.6 females vs 4.1 males $(P=0.02)$; and the depression subscale was 8.2 females vs 5.1 males $(P=0.04)$. There was no significant difference in resident DASS-21 scores at baseline with regard to age, children at home, race, or specialty (Table 1). PGY1 residents trended toward more stress compared to more advanced residents, although this difference was not statistically significant $(P=0.34)$.

Burnout scores on the initial (baseline) survey were in the abnormal range for most residents, both with respect to exhaustion (38/47 residents) and disengagement (37/47 residents), using the OLBI questionnaire. There was no difference in resident OLBI scores at baseline with regard to age, children at home, residency year, race, or specialty. Although there was also no difference in total mean OBLI scores between male and female residents, females had higher exhaustion subscores (2.6, female vs 2.4, male; $P=0.03$ ).

Results from the psychological well-being research using MAAS and CFQ indicated that there was no difference in resident scores at baseline with regard to age, children, race, or
A Initial and follow-up $(n=30)$
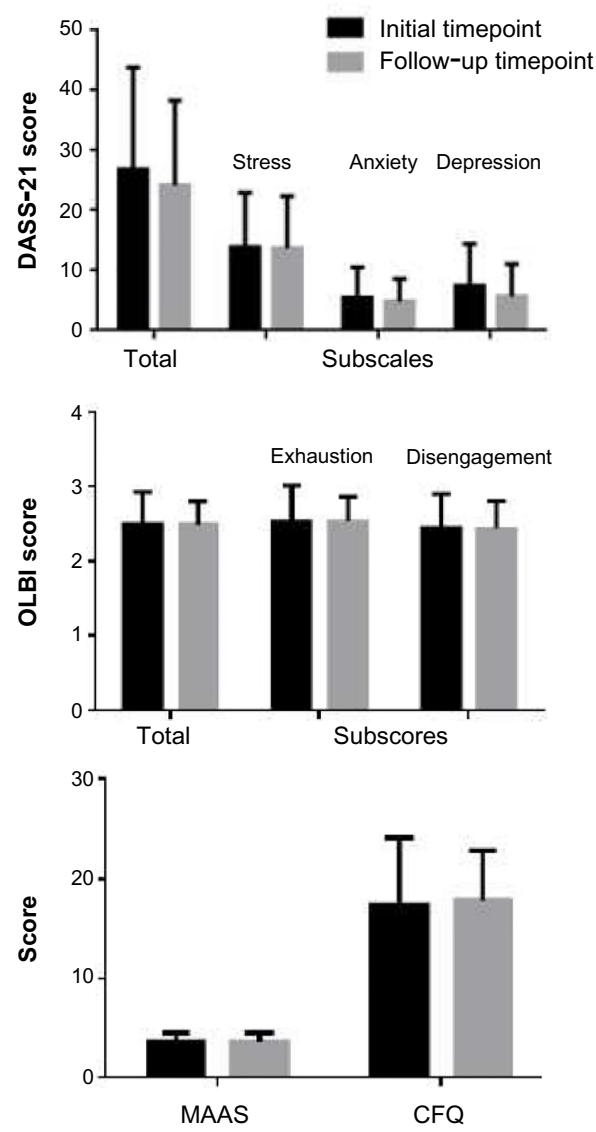

\section{B Longer follow-up $(n=7)$}
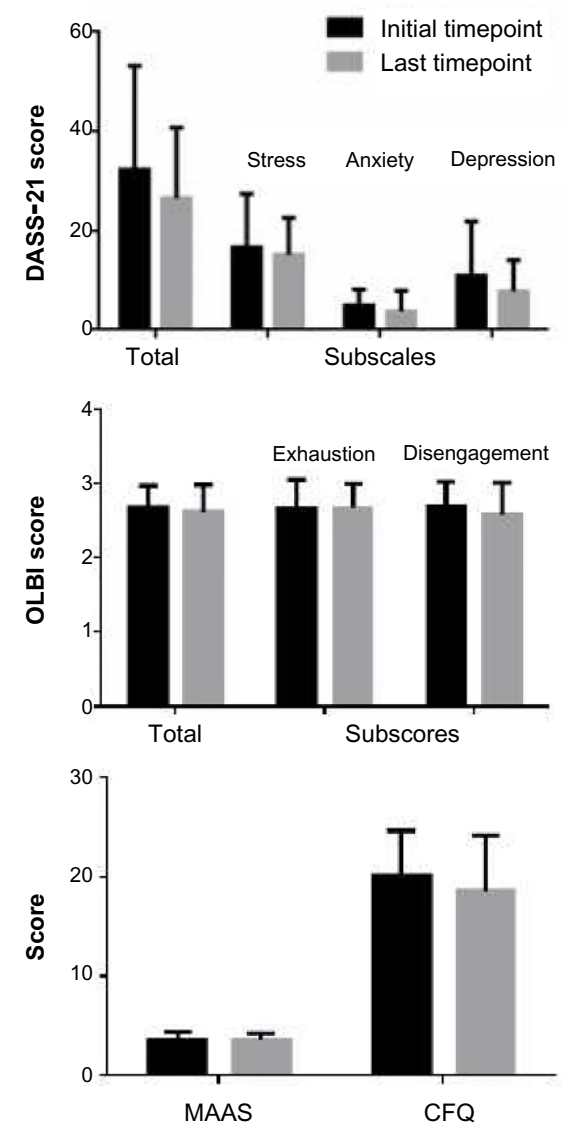

Figure I Stress, burnout, mindful awareness, and cognitive failure scores in residents before and after the mindfulness-based resilience intervention.

Notes: (A) Thirty residents completed both the initial and follow-up paper-based surveys. Mean scores for the DASS-2I (with stress, anxiety, and depression subscales), OLBI (with exhaustion and disengagement subscores), MAAS, and CFQ are shown. There is no significant difference in the scores before and just after the intervention. (B) Seven residents completed the initial paper-based survey ("Initial timepoint"), the follow-up paper-based survey (just following the intervention), and the online survey ( I month after the intervention, “Last timepoint"). Mean scores for the DASS-2I (with stress, anxiety, and depression subscales), OLBI (with exhaustion and disengagement subscores), MAAS, and CFQ scores are shown for the initial survey and the final survey ( I month after the intervention). Although there is no significant difference in the scores before and just after the intervention, there appears to be a trend toward lower stress scores at the longer follow-up timepoint. Error bars, standard deviation. Abbreviations: DASS-2I, Depression-Anxiety-Stress Scale, 21 -question version; OLBI, Oldenburg Burnout Inventory; MAAS, Mindful Attention Awareness Scale; CFQ, Cognitive Failures Questionnaire. 
specialty (Table 1). Female residents had higher CFQ scores, or more perceived failures (19.9, female vs 14.8 , male; $P=0.01)$. Although not statistically significant, residents in earlier years of training had a trend of higher CFQ scores than those in their later years (22.9 for PGY1 vs 15.1 for PGY4; $P=0.25$ ).

\section{Perceived stress correlates with stress and burnout instrument scores}

At baseline, most residents indicated that residency was at least "somewhat stressful" (Table 2). Higher perceived levels of stress correlated with higher stress scores on the DASS-21 for the total score $(P=0.01)$, stress subscore $(P=0.01)$, and depression subscore $(P=0.02)$. Higher perceived levels of stress also correlated with more burnout: higher OLBI total $(P=0.02)$, higher OLBI exhaustion subscale $(P=0.01)$, and higher perceived failures $(P=0.03)$. Higher perceived stress levels correlated with lower scores on the MAAS $(P=0.02)$, meaning that lower levels of mindful-awareness were seen in residents with higher perceived stress.

\section{No short-term effect on stress and burnout in overall sample}

Thirty residents completed the initial survey and the survey following the final session. Over this time period, there was no significant change in stress, burnout, mindful- awareness, or cognitive failures (Figure 1A). Seven of the 30 residents completed a follow-up survey 1 month after the final session (Figure 1B). At this later timepoint, there was a trend toward lower scores, although this trend was not statistically significant.

\section{Subset analysis for target population}

In the subset analysis (Figure 2), there was a trend for female residents and $\mathrm{PGY} 1$ and $\mathrm{PGY} 2$ residents to have a reduction in DASS-21 scores and the CFQ score, comparing scores from before and after the intervention. For those residents who perceive residency to be stressful, there was a trend for higher perceived residency stress to correlate with a reduction in stress and burnout after our intervention (Figure 3). Overall, the trends did not reach statistical significance, except in the case of the OLBI disengagement subscale, which was statistically significant $(P=0.03)$.

\section{Discussion}

Resident stress and burnout were evaluated both before and after a mindfulness-based resilience intervention. At baseline, residents overall had normal levels of stress, but females had significantly higher stress scores than males, and a majority of residents had high levels of exhaustion and disengagement as measured by the OLBI, suggesting a high prevalence of

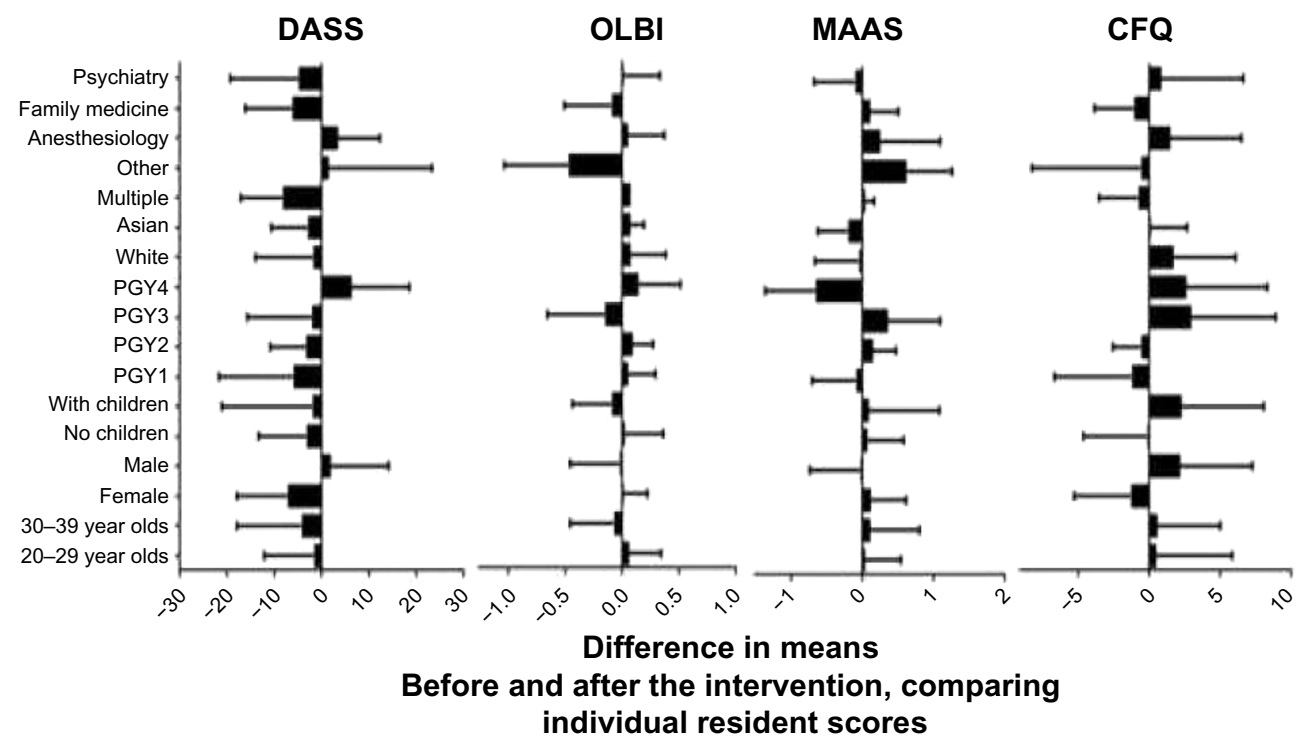

Figure 2 Trends in demographic characteristics and effect of the intervention.

Notes: For each resident, the difference in means was calculated for each instrument by subtracting the follow-up score from the initial score. For example, the total DASS21 score from the follow-up survey was subtracted from the total DASS-2I score from the initial survey. A negative difference in means indicates that the score decreased due to the intervention; this decrease was the goal of the intervention with regard to the stress and burnout instruments (DASS-2I and OLBI). A positive difference would indicate that the score increased due to the intervention; an increasing score on the MAAS would indicate greater mindful awareness after the intervention. The difference in means is graphed for each demographic group. The results suggest that female residents and residents early in the program may benefit most from the intervention. Error bars, standard deviation.

Abbreviations: DASS-2I, Depression-Anxiety-Stress Scale, 2I-question version; OLBI, Oldenburg Burnout Inventory; MAAS, Mindful Attention Awareness Scale; CFQ, Cognitive Failures Questionnaire. 


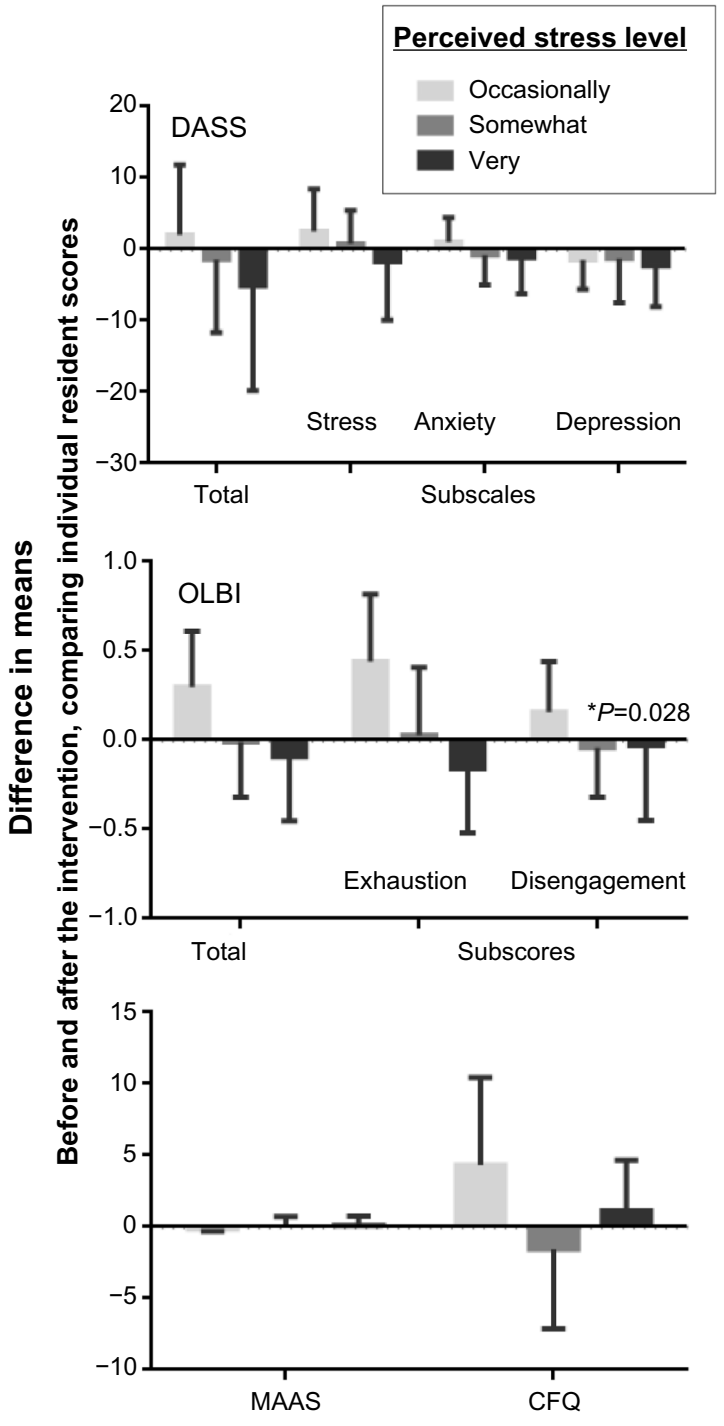

Figure 3 Trends in perceived stress and effect of the intervention.

Notes: For each resident, the difference in means was calculated for each instrument by subtracting the follow-up score from the initial score. For example, the total DASS-2I score from the follow-up survey was subtracted from the total DASS21 score from the initial survey. A negative difference in means indicates that the score decreased due to the intervention; this result was the goal of the intervention with regard to the stress and burnout instruments (DASS-2I and OLBI). A positive difference would indicate that the score increased due to the intervention; an increasing score on the MAAS would indicate greater mindful awareness after the intervention. The difference in means is graphed for the groups of residents who perceived residency to be occasionally stressful, somewhat stressful, and very stressful. The results suggest the trend that residents who perceive residency to be more stressful had decreasing stress and burnout after the intervention. All $P$-values were $>0.05$, except where indicated, for the OLBI disengagement subscore. Error bars, standard deviation. "P-value based on Kruskal-Wallis test.

Abbreviations: DASS-2I, Depression-Anxiety-Stress Scale, 21 -question version; OLBI, Oldenburg Burnout Inventory; MAAS, Mindful Attention Awareness Scale; CFQ, Cognitive Failures Questionnaire.

burnout. Our results also suggested that residents knew when they were stressed, since perceived stress correlated with the validated instrument scores. Mindful-awareness was inversely correlated to perceived stress in the residents in the study. The mindfulness-based intervention did not affect stress and burnout in the short-term; however, there was a trend at later timepoints toward lower scores in stress and burnout, suggesting that longer follow-up may be helpful.

Burnout in residents has been documented by previous studies. A recent large national study examining medical trainees and physicians early in their careers found that they were more likely to experience burnout than the general population and that being a resident or fellow was associated with greater odds of burnout. ${ }^{23}$ As in our current study, these investigators also found that female physicians had higher odds of having high fatigue and depression than male physicians. This finding may be due a greater concern about work-life balance among females, ${ }^{24}$ who perhaps also experienced additional demands outside of residency. Female residents may feel time pressure with regard to family planning at the time of residency as compared to their male counterparts, since residency frequently occurs in the third decade of life. Another research group found that female residents had an increased frequency of emotional exhaustion as compared to males, and also reported that emotional exhaustion was more common among those residents in a lower PGY. ${ }^{25} \mathrm{~A}$ third research group also found that residents in their first year of residency had higher rates of burnout, and similar to our current study, found no significant difference between specialties ${ }^{26}$ The literature reports a wide range of burnout $(27 \%-75 \%),{ }^{27}$ possibly due to differences in instruments used; in our present study, the rate of burnout was approximately $80 \%$ ( $81 \%$, exhaustion subscore; $79 \%$, disengagement subscore).

Several other research groups have studied stress reduction programs offered during residency. One group conducted a half-day workshop for medicine and pediatric residents that focused on relationships, prioritization of obligations, and stamina and outlook skills. ${ }^{28}$ They noted improvements in burnout: emotional exhaustion scores improved and depersonalization scores did not deteriorate as much as the nonintervention group. Another research group studied family practice residents and utilized the Respiratory One Method (a meditation practice combining breathing and repetition) for stress reduction, with results showing a positive effect on emotional exhaustion. ${ }^{29}$ In both these studies, ${ }^{28,29}$ only residents who were released from clinical duties were able to participate in the intervention group, potentially creating bias and limiting generalizability. A third research group conducted a randomized, controlled study among anesthesia residents using the "Coping with Work and Family Stress" intervention; residents who participated in the intervention reported less family stress, increased support at work, and less anxiety. ${ }^{30}$

Our present study was prospective but lacked a control group. It was designed to detect differences in stress metrics 
on residents before and after the intervention, but was not powered to detect differences in the subgroups. The study was done at a single institution and with residents sourced from a limited number of medical specialties, potentially limiting the generalizability of the results. Also, this study was conducted with the support of the various program directors during protected didactic time, with $43 \%$ overall participation; however, only residents without clinical duties were able to participate in the study, which may underrepresent residents with greater clinical demands. The suboptimal completion rate may be due to residents' lack of time, a high number of competing email requests and demands, lack of study compensation, lack of interest in the topic, or length of the survey. In addition, to protect the confidentiality of the training sessions and the anonymity of the study participants, attendance was not taken during the sessions; thus, the completion rate likely underestimates the true study participation rate. Due to the voluntary nature of the intervention, selection bias may exist, because residents who were not feeling stress may not have come to the sessions. Due to complex programmatic demands, study duration was variable, which may have limited the effect of the intervention. Additionally, validated instruments were used for stress, burnout, and mindful-awareness, but a subset of the CFQ including the questions most pertinent to residents was also used. This methodology was used to maintain an overall survey length that was acceptable to the study participants, but may have limited the utility of this instrument. Finally, we did not find a significant short-term effect on stress and burnout after our resilience training, which was shortened to feasibly be offered during regular didactic time; a longer, more intensive training may be more effective.

\section{Conclusion}

The present study found that medical residents have high levels of burnout and that this burnout correlates with selfreported stress levels. There was no significant short-term change in stress and burnout after the intervention; however, our findings suggested that there may be benefit to targeting resiliency training to specific residents, including females, PGY1 and PGY2 residents, and residents who perceive residency to be stressful.

\section{Acknowledgment}

This work was funded by a grant to KK from the Gold Foundation, which provided salary support for KK for development of the mindfulness-based intervention.

\section{Disclosure}

The authors report no conflicts of interest in this work.

\section{References}

1. Resident Services Committee, Association of Program Directors in Internal Medicine. Stress and impairment during residency training: strategies for reduction, identification, and management. Ann Intern Med. 1988;109(2):154-161.

2. Levey RE. Sources of stress for residents and recommendations for programs to assist them. Acad Med. 2001;76(2):142-150.

3. Thomas NK. Resident burnout. JAMA. 2004;292(23):2880-2889.

4. Schaufeli WB, Buunk BP. Professional burnout. In: Schabracq MJ, Winnubst JAM, Cooper CL, editors. Handbook of Work and Health Psychology. New York: Wiley; 1996:513-527.

5. Maslach C, Jackson SE. Maslach Burnout Inventory: Manual Research Edition. Palo Alto: Consulting Psychologists Press; 1986.

6. Maslach C, Jackson SE, Leiter MP. Maslach Burnout Inventory Manual. 3rd ed. Palo Alto: Consulting Psychologists Press; 1996.

7. Kang EK, Lihm HS, Kong EH. Association of intern and resident burnout with self-reported medical errors. Korean J Fam Med. 2013;34(1): 36-42.

8. West CP, Huschka MM, Novotny PJ, et al. Association of perceived medical errors with resident distress and empathy: a prospective longitudinal study. JAMA. 2006;296(9):1071-1078.

9. Shanafelt TD, Bradley KA, Wipf JE, Back AL. Burnout and selfreported patient care in an internal medicine residency program. Ann Intern Med. 2002;136(5):358-367.

10. Prins JT, Hoekstra-Weebers JE, Gazendam-Donofrio SM, et al. Burnout and engagement among resident doctors in the Netherlands: a national study. Med Educ. 2010;44(3):236-247.

11. Paulson S, Davidson R, Jha A, Kabat-Zinn J. Becoming conscious: the science of mindfulness. Ann NY Acad Sci. 2013;1303:87-104.

12. Kabat-Zinn J. Wherever You Go, There You Are. New York: Hyperion Publishing; 1994.

13. Finkelstein C, Brownstein A, Scott C, Lan YL. Anxiety and stress reduction in medical education: an intervention. Med Educ. 2007;41(3):258-264.

14. Rosdahl JA, Kingsolver KO. Mindfulness training to increase resilience and decrease stress and burnout in ophthalmology residents: a pilot study. Invest Ophthalmol Vis Sci. 2014;55(13):5579-5579.

15. Harris PA, Taylor R, Thielke R, Payne J, Gonzalez N, Conde JG. Research electronic data capture (REDCap) - a metadata-driven methodology and workflow process for providing translational research informatics support. J Biomed Inform. 2009;42(2):377-381.

16. Lovibond SH, Lovibond PF. Manual for the Depression Anxiety Stress Scales. 2nd ed. Sydney: Psychology Foundation; 1995.

17. Lovibond PF, Lovibond SH. The structure of negative emotional states: comparison of the Depression Anxiety Stress Scales (DASS) with the Beck Depression and Anxiety Inventories. Behav Res Ther. 1995;33(3):335-343.

18. Halbesleben JRB, Demerouti E. The construct validity of an alternative measure of burnout: investigating the English translation of the Oldenburg Burnout Inventory. Work Stress. 2005;19(3):208-220.

19. Demerouti E, Bakker AB, Vardakou I, Kantas A. The convergent validity of two burnout instruments: a multitrait-multimethod analysis. Eur J Psychol Assess. 2003;19(1):12-23.

20. Brown KW, Ryan RM. The benefits of being present: mindfulness and its role in psychological well-being. J Pers Soc Psychol. 2003;84(4):822-848.

21. Broadbent DE, Cooper PF, FitzGerald P, Parkes KR. The Cognitive Failures Questionnaire (CFQ) and its correlates. Br J Clin Psychol. 1982;21(Pt 1):1-16.

22. Peterson U, Demerouti E, Bergström G, Åsberg M, Nygren Å. Work characteristics and sickness absence in burnout and nonburnout groups: a study of Swedish health care workers. Int J Stress Manag. 2008;15(2):153-172. 
23. Dyrbye LN, West CP, Satele D, et al. Burnout among US medical students, residents, and early career physicians relative to the general US population. Acad Med. 2014;89(3):443-451.

24. Hendrie HC, Clair DK, Brittain HM, Fadul PE. A study of anxiety/ depressive symptoms of medical students, house staff, and their spouses/ partners. J Nerv Ment Dis. 1990;178(3):204-207.

25. West CP, Shanafelt TD, Kolars JC. Quality of life, burnout, educational debt, and medical knowledge among internal medicine residents. JAMA. 2011;306(9):952-960.

26. Martini S, Arfken CL, Churchill A, Balon R. Burnout comparison among residents in different medical specialties. Acad Psychiatry. 2004;28(3):240-242.
27. Ishak WW, Lederer S, Mandili $\mathrm{C}$, et al. Burnout during residency training: a literature review. J Grad Med Educ. 2009;1(2):236-242.

28. McCue JD, Sachs CL. A stress management workshop improves residents' coping skills. Arch Intern Med. 1991;151(11):2273-2277.

29. Ospina-Kammerer V, Figley CR. An evaluation of the Respiratory One Method (ROM) in reducing emotional exhaustion among family physician residents. Int J Emerg Ment Health. 2003;5(1):29-32.

30. Saadat H, Snow DL, Ottenheimer S, Dai F, Kain ZN. Wellness program for anesthesiology residents: a randomized, controlled trial. Acta Anaesthesiol Scand. 2012;56(9):1130-1138.

\section{Publish your work in this journal}

Advances in Medical Education and Practice is an international, peerreviewed, open access journal that aims to present and publish research on Medical Education covering medical, dental, nursing and allied health care professional education. The journal covers undergraduate education, postgraduate training and continuing medical education including emerging trends and innovative models linking education, research, and health care services. The manuscript management system is completely online and includes a very quick and fair peer-review system. Visit http://www.dovepress.com/testimonials.php to read real quotes from published authors.

Submit your manuscript here: http://www.dovepress.com/advances-in-medical-education-and-practice-journal 